\title{
Adequate sleep to improve the treatment of obesity
}

\author{
Jean-Philippe Chaput PhD, Angelo Tremblay PhD
}

$\mathrm{E}$ nergy restriction combined with increased physical activity is widely used to induce weight loss and reduce the health risks associated with excess fat. However, individual responses to typical weight-loss programs can vary greatly, emphasizing the need to adopt more personalized approaches. Less considered factors may explain the failure of traditional treatments. Among the behavioural factors that have been shown to impede weight loss, insufficient sleep is gaining attention and recognition.

Chronic sleep restriction is pervasive in modern societies, ${ }^{1}$ and there is robust evidence supporting the role of reduced sleep as contributing to the current obesity epidemic. ${ }^{2}$ New studies provide evidence that insufficient sleep enhances hedonic stimulus processing in the brain underlying the drive to consume food; thus, insufficient sleep results in increased food intake. ${ }^{3}$ In addition, lack of sleep has been reported to decrease plasma leptin levels, increase plasma ghrelin and cortisol levels, alter glucose homeostasis and activate the orexin system, all of which affect the control of appetite and might compromise the efficacy of dietary interventions. ${ }^{4}$

Further support for the importance of sleep comes from a recent crossover study involving overweight adults. ${ }^{5}$ Participants were randomly assigned to sleep either 5.5 hours or 8.5 hours each night for 14 days, in conjunction with moderate caloric restriction (average daily deficit of about $680 \mathrm{kcal}$ ) in a laboratory setting. ${ }^{5}$ Compared with participants who slept 8.5 hours per night, participants who slept only 5.5 hours lost $55 \%$ less body fat and $60 \%$ more fat-free mass; they also had less-favourable changes in metabolic hormone levels and substrate and energy use. Despite a loss of about $3 \mathrm{~kg}$ of body weight during each treatment, total energy loss was twice as high in the 8.5-hour group (1039 kcal/d) than in the 5.5 -hour group $(573 \mathrm{kcal} / \mathrm{d})$. Thus, study participants defended their energy balance more vigorously (i.e., they conserved energydense fat at the expense of a greater loss of lean body mass) when they did not get enough sleep. In addition, participants in the shorter sleep group perceived greater hunger (accompanied by higher ghrelin levels) than participants in the longer sleep group. Given that higher ghrelin concentrations may facilitate the retention of fat, and increased hunger could compromise adherence to caloric restriction, these mechanisms may help explain why insufficient sleep could compromise the efficacy of common dietary interventions.

In support of these novel findings, we recently assessed in a real-life setting whether adequate sleep might be an important factor in successful weight loss. We looked at the amount of fat loss in 123 adults who were overweight or obese and undergoing moderate caloric restriction supervised by a dietician for 17 weeks. We found that both total sleep time and sleep quality at baseline predicted loss of fat mass. ${ }^{6}$ Other recent studies have yielded similar results. Among 245 women enrolled in a 6-month weight-loss program, better subjective sleep quality increased the likelihood of successful weight loss by $33 \%$, as did sleeping more than 7 hours per night. ${ }^{7}$

Nonpharmacologic and behaviour-based interventions were used to increase sleep duration in a prospective randomized controlled trial to measure the effects of extending sleep on body weight in short-duration sleepers with obesity. ${ }^{8}$ Preliminary observations suggest that the participants in the intervention group reported more willingness to exercise and fewer cravings for sweet and salty foods during the evening than participants in the control group. ${ }^{8}$

In a longitudinal observational study, we recently investigated whether a favourable change in sleep duration over 6 years could affect objective indicators of adiposity in adults. ${ }^{9}$ That is, we tested whether a spontaneous change in sleep duration (from $\leq 6 \mathrm{~h} / \mathrm{d}$ to $7-8 \mathrm{~h} / \mathrm{d}$ ) can influence adiposity. We saw that shifting sleep

\section{- KEY POINTS}

- Increasing evidence supports the role of insufficient sleep in contributing to obesity.

- Lack of sufficient sleep may compromise the efficacy of common weight-loss interventions.

- Shifting sleep duration from a short to a healthier amount of time is associated with attenuated gain in fat mass.

- Addressing sleep for weight management has recently been endorsed by the Canadian Obesity Network.
Competing interests: None declared.

This article has been peer reviewed.

Correspondence to: Jean-Philippe Chaput, jpchaput@cheo.on.ca

CMAJ 2012. DOI:10.1503 /cmaj.120876 
duration from a short to a healthier amount of time is associated with an attenuated gain in fat mass $(2.4 \mathrm{~kg}$ less in people who increased their sleep duration compared with those who maintained their short sleep duration).

The Canadian Obesity Network recently launched a set of practitioner tools - the 5As of Obesity Management (ask, assess, advise, agree and assist) - that highlight the importance of addressing sleep for weight management. ${ }^{10}$ Techniques for screening and diagnosing common sleep/wake disorders can be of use to clinicians. ${ }^{11}$ In particular, the Pittsburgh Sleep Quality Index is a good tool for inquiring about the quality and duration of sleep. ${ }^{12}$ In addition, clinicians should remember that the association between inadequate sleep and obesity can be confounded by factors such as stress, depression, psychosocial problems, chronic illness and the use of hypnotic agents.

Future research should try to identify waking activities under discretionary control that could be replaced with increased time spent sleeping (e.g., television viewing in the evening, start times for work and high school in the morning, distance to work) and testing the effectiveness of interventions for achieving additional sleep (e.g., moving prime-time television programs to earlier in the evening).

Successful weight management is complicated, and a good understanding of the root causes of weight gain and barriers to weight management is essential to success. The solution is not as simple as "eat less, move more, sleep more." However, an accumulating body of evidence suggests that sleeping habits should not be overlooked when prescribing a weight-reduction program to a patient with obesity. Sleep should be included as part of the lifestyle package that traditionally has focused on diet and physical activity.

\section{References}

1. Matricciani L, Olds T, Petkov J. In search of lost sleep: secular trends in the sleep time of school-aged children and adolescents. Sleep Med Rev 2012;16:203-11.

2. Cappuccio FP, Taggart FM, Kandala NB, et al. Meta-analysis of short sleep duration and obesity in children and adults. Sleep 2008;31:619-26

3. Chaput JP, Tremblay A. Insufficient sleep as a contributor to weight gain: an update. Curr Obes Rep. In press.

4. Leproult R, Van Cauter E. Role of sleep and sleep loss in hormonal release and metabolism. Endocr Dev 2010;17:11-21.

5. Nedeltcheva AV, Kilkus JM, Imperial J, et al. Insufficient sleep undermines dietary efforts to reduce adiposity. Ann Intern Med 2010;153:435-41.

6. Chaput JP, Tremblay A. Sleeping habits predict the magnitude of fat loss in adults exposed to moderate caloric restriction. Obes Facts 2012;5:561-6.

7. Thomson CA, Morrow KL, Flatt SW, et al. Relationship between sleep quality and quantity and weight loss in women participating in a weight-loss intervention trial. Obesity (Silver Spring) 2012;20:1419-25.

8. Cizza G, Marincola P, Mattingly M, et al. Treatment of obesity with extension of sleep duration: a randomized, prospective, controlled trial. Clin Trials 2010;7:274-85.

9. Chaput JP, Després JP, Bouchard C, et al. Longer sleep duration associates with lower adiposity gain in adult short sleepers. Int $J$ Obes (Lond) 2012;36:752-6.

10. 5As of obesity management. Edmonton (AB): Canadian Obesity Network; 2012. Available: www.obesitynetwork.ca/5As (accessed 2012 Aug. 16)

11. Blythe J, Doghramji PP, Jungquist CR, et al. Screening and treating patients with sleep/wake disorders. JAAPA 2009 (Sleep Suppl):1-17.

12. Buysse DJ, Reynolds CF, Monk TH, et al. The Pittsburgh Sleep Quality Index: a new instrument for psychiatric practice and research. Psychiatry Res 1989;28:192-213.

Affiliations: From the Healthy Active Living and Obesity Research Group (Chaput), Children's Hospital of Eastern Ontario Research Institute, Ottawa, Ont.; and the Department of Kinesiology (Tremblay), Laval University, Québec, Que.

Contributors: Both of the authors contributed to the conception and design of the article. Jean-Philippe Chaput drafted the article, and Angelo Tremblay revised it for important intellectual content. Both of the authors approved the final version of the manuscript submitted for publication. 